\title{
Sydney observatory Galactic survey
}

\section{Potential for detection of extreme disk-crossing stars ${ }^{\star}$}

\author{
A. Fresneau ${ }^{1}$, A. E. Vaughan ${ }^{2}$, and R. W. Argyle 3 \\ 1 Observatoire astronomique, Université Louis Pasteur, CNRS, 11 rue de l'Université, 67000 Strasbourg, France \\ e-mail: fresneau@astro.u-strasbg.fr \\ 2 Department of Physics, Macquarie University, Sydney 2109, New South Wales, Australia \\ e-mail: alanv@ics.mq.edu.au \\ 3 Institute of Astronomy, Madingley Road, Cambridge, CB3 0HA, UK \\ e-mail: rwa@ast.cam.ac.uk
}

Received 23 January 2007 / Accepted 2 May 2007

ABSTRACT

\begin{abstract}
Context. First Epoch astrometric and radiometric information of a survey in the optical along the Galactic fourth quadrant is archived for further analysis in the time-domain.

Aims. A photographic survey of $1.9 \times 10^{6}$ stars down to $B$-magnitude $14 \pm 0.5$ along the galactic meridian $l=(330 \pm 5)^{\circ},-64^{\circ} \leq b \leq$ $-7^{\circ}$ and along the galactic equator $272^{\circ} \leq l \leq 341^{\circ},|b| \leq 7^{\circ}$, is provided from century old astrographic plates of Sydney Observatory. Stellar candidates to investigate the Galaxy disk kinematics up to a distance of $1 \mathrm{kpc}$ from the Sun are tentatively selected.

Methods. The early data are combined with late epoch observations into a set of $4.8 \times 10^{5}$ stars with significant proper motion $\mu \geq 0.005^{\prime \prime} \mathrm{yr}^{-1}$ and approximate color $B-V$. The selection effects and the kinematical bias on the stellar system are investigated. F-G stellar candidates with a mean transverse velocity dispersion of $45 \mathrm{~km} \mathrm{~s}^{-1}$ are suggested.

Results. The spin rate of $\frac{1}{2}^{\circ} \mathrm{pc}^{-1}$ of the velocity ellipsoid along its long axis is observed over half a thickness of $25 \mathrm{pc}$ of the galactic disk for stars at an estimated distance $D \leq 300 \mathrm{pc}$ from the Sun.

Conclusions. A catalogue of $4.8 \times 10^{5}$ stars is provided for further investigation of the galactic disk heating by transient spiral wave or by GMC encounter.
\end{abstract}

Key words. surveys - stars: kinematics

\section{Retrospective of early astrographic surveys}

First epoch observations used in the proper motion (p.m.) determination of faint stars observed in the Tycho- 2 catalogue are based on short exposure plates observed for the Astrographic Catalog (AC) at the beginning of the 20th century over a field of view of $2^{\circ} \times 2^{\circ}$ for each of the $4 \times 10^{4} \mathrm{AC}$ plates at a scale of $1^{\prime} \mathrm{mm}^{-1}$, proving that photographic observations from the past were indeed durable even at the time of space astrometry (Urban et al. 1998). In order to check the quality of the old plates with a fast measuring machine like APM, used for instance to scan large Schmidt plates (Evans 1992), a set of 104 short exposure plates dating from the late 19th century was selected from the plate vault of Sydney Observatory, archived at Macquarie University.

The double exposure technique used in the astrophotographic survey is shown in Fig. 1: stars are identified in the image inventory of a plate scanned with APM by the combination of double images. Along with the $x, y$ plate mean coordinates (in $\mathrm{mm}$ ) and their errors $\Delta x, \Delta y$ (in microns), an indication of short term magnitude variability $\Delta m$, is also computed for each star: this information is not available from the AC measurements but is usable to search for flare stars (Fresneau et al. 2001). The $(\xi, \eta)$ plate coordinates (in arcmin) for the Tycho-2 stars

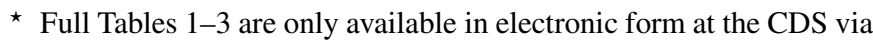
anonymous ftp to cdsarc.u-strasbg.fr (130.79.128.5) or via http://cdsweb.u-strasbg.fr/cgi-bin/qcat?]/A+A/469/1221 are derived from the equatorial coordinates $\alpha, \delta$ at the epoch of $\mathrm{AC}$ observations as the result of a gnomonic projection around the direction $\left(\alpha_{0}, \delta_{0}\right)$ and over a field of view of $2^{\circ} \times 2^{\circ}$ at a plate scale of $1^{\prime} \mathrm{mm}^{-1}$ (König 1962): $\frac{\xi}{f}=\frac{\cos \delta \sin \left(\alpha-\alpha_{0}\right)}{\sin \delta \sin \delta_{0}+\cos \delta \cos \delta_{0} \cos \left(\alpha-\alpha_{0}\right)}$ and $\frac{\eta}{f}=\frac{\sin \delta \cos \delta_{0}-\cos \delta \sin \delta_{0} \cos \left(\alpha-\alpha_{0}\right)}{\sin \delta \sin \delta_{0}+\cos \delta \cos \delta_{0} \cos \left(\alpha-\alpha_{0}\right)}$ (with $f=3438$ ).

The external orientation of the telescope relatively to the sky $\left(\Delta \alpha_{0}, \Delta \delta_{0}\right)$ and the internal calibration of the plate scale $(\Delta f)$ are derived from the Turner equations (Turner 1893) fitted by a linear adjustment between $\xi, \eta$ of the Tycho-2 stars (in arcmin) and measured $(x, y)$ coordinates (in $\mathrm{mm}$ ):

$\xi=A x+B y+C+D x^{2}+E x y+F y^{2}$,

$\eta=A^{\prime} x+B^{\prime} y+C^{\prime}+D^{\prime} x^{2}+E^{\prime} x y+F^{\prime} y^{2},\left(A \simeq B^{\prime} \simeq 1, B \simeq-A^{\prime} \simeq 0\right)$.

The quality of the best linear unbiased estimation of the set of 12 plate constants $\left(A, B, C, D, E, F\right.$ and $\left.A^{\prime}, B^{\prime}, C^{\prime}, D^{\prime}, E^{\prime}, F^{\prime}\right)$ after the weighted least squares fit is measured by the rms error $\sigma_{\mathrm{T}}$ between the computed and observed equatorial coordinates of the set of Tycho- 2 stars (a couple of hundreds per $2^{\circ} \times 2^{\circ}$ field of view). The same stars are also used to derive a calibration curve between $B$-magnitude of Tycho- 2 stars and the radiometric stellar image density measured by APM.

The inventory of all stellar images can also be adjusted to the GSC version 1.2 (Lasker et al. 1990). In that case, the number of stars (a couple of thousands) used in the calibration is about ten times larger, especially for the deep plates close to the fainter magnitude limit provided by the GSC survey (Fresneau 1985). 


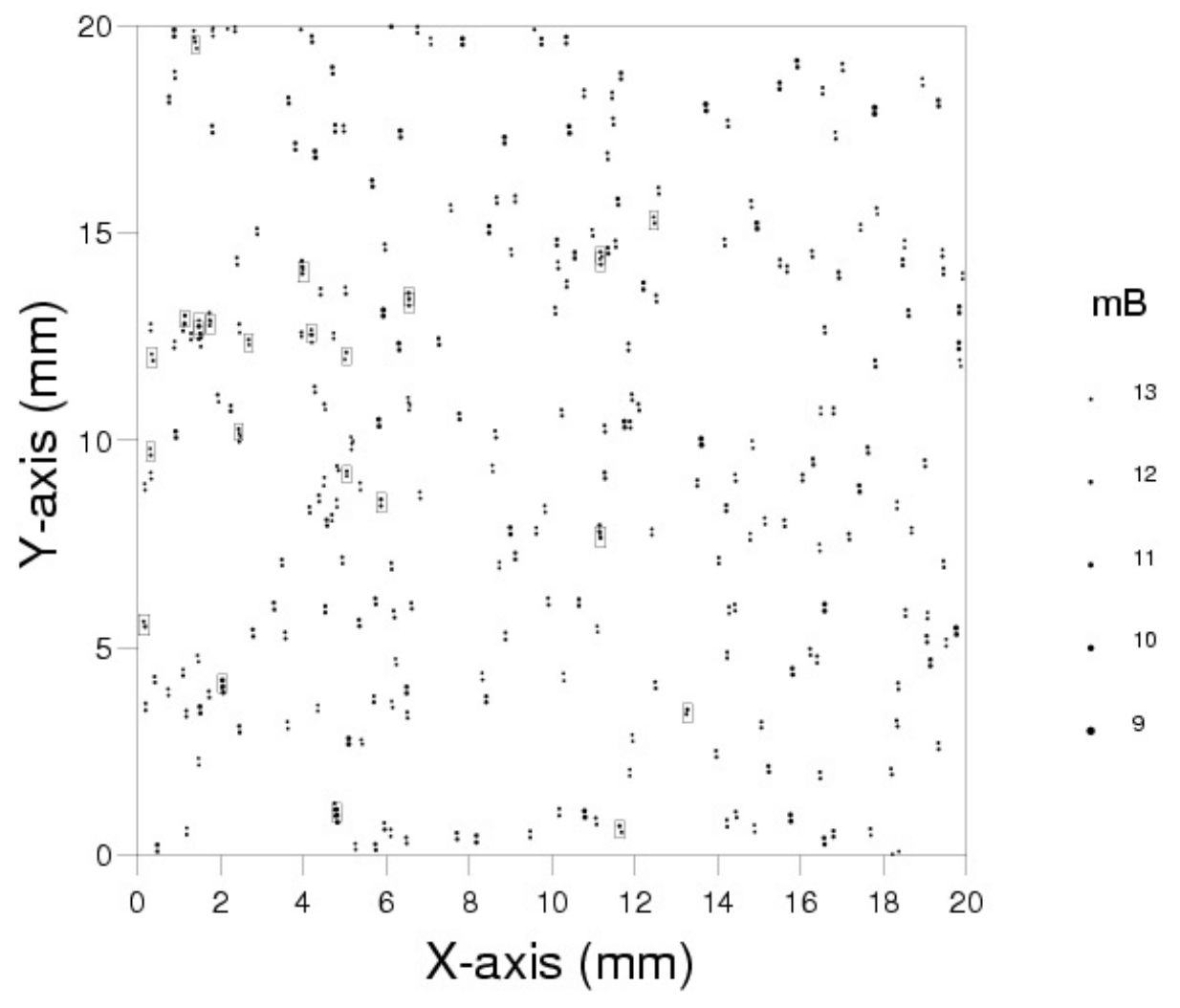

Fig. 1. The legacy of the all-sky astrographic program is a set of $4 \times 10^{4}$ one century old photographic plates with a field of view of $2^{\circ} \times 2^{\circ}$ at a scale of $1^{\prime} \mathrm{mm}^{-1}$. Double exposures segregate stellar images from plate defect. Stars with significant short term radiometric variability are plotted in a rectangle.

The p.m. components $\mu_{x}, \mu_{y}$ (in ' $\mathrm{yr}^{-1}$ ) of the GSC stars are computed according to the relations $\mu_{x}=\frac{\Delta \xi}{\Delta t}, \mu_{y}=\frac{\Delta \eta}{\Delta t}$, with $\Delta \xi=\xi-A x-B y-C-D x^{2}-E x y-F y^{2}$ and $\Delta \eta=\eta-A^{\prime} x-$ $B^{\prime} y-C^{\prime}-D^{\prime} x^{2}-E^{\prime} x y-F^{\prime} y^{2}$, and $\Delta t$ the difference between the epoch of the AAO Schmidt telescope observations (Table III of Lasker et al. 1990) and the epoch of the AC observations. The set of 12 plates constants is estimated after 3 iterations of a weighted least squares fit providing a rms error $\sigma_{\mathrm{G}}$. Stars with total proper motion $\mu \geq 3 \sigma_{\mathrm{G}}$ are ignored at each step in the estimation of the plate constants. The set of 12 plate constants fitting the Turner equations is unbiased if the residuals $\Delta \xi, \Delta \eta$ of the final group of reference stars are of mean value zero, Gaussian and independent (Gauss-Markoff theorem, Babu \& Feigelson 1996). A half-normal plot (Lutz \& Upgren 1980) of these residuals was used to define the significant level $\sigma_{\mu}$ of the total p.m. $\mu$ and we observed $\sigma_{\mu} \approx 3 \sigma_{\mathrm{G}}$, with a final percentage of stars fitting a normal distribution on the order of $75 \%$ of the total number of stars on a plate. As a result of this procedure, a set of $4.8 \times 10^{5}$ stars with significant p.m. $\mu \geq(0.015 \pm 0.005)^{\prime \prime} \mathrm{yr}^{-1}$ is extracted out of a survey of $1.9 \times 10^{6}$ stars and is considered as the set of foreground stars. The mean motion $\mu_{0}$ of the set of background stars is on the order of $\mu_{0} \leq(0.002 \pm 0.001)^{\prime \prime} \mathrm{yr}^{-1}$ (Vyssotsky 1954).

Table 1 lists the set of 104 short exposure plates as early as 1893 that were measured in 2002 with APM for checking the measurements published in the 1920's by Sydney Observatory (and used in AC2000). The data set is available on-line ${ }^{1}$, with the metric $(\Delta x, \Delta y$ in microns) and radiometric $(\Delta m)$ errors estimated for each star detected on these plates, with their $(x, y)$ plate coordinates in $\mathrm{mm}$, the $B$-magnitude $(m)$, equatorial coordinates ( $\alpha, \delta$ at equinox and equator 2000, but at epoch of the plate)

\footnotetext{
${ }^{1}$ See ftp://cdsarc.u-strasbg.fr/incoming/fresneau/ac
}

and their errors $(\Delta \alpha, \Delta \delta$ in ") derived from the $6 \times 6$ variancecovariance matrix of the 6 plate constants in each coordinate estimated in the least squares solution of the Turner equations (Eichhorn \& Williams 1963). Table 1 identifies the plate center coordinates $\left(\alpha_{0}, \delta_{0}\right.$ at equinox and equator 1900), the epoch of observations, the quality of the adjustment to Tycho-2 catalogue $\sigma_{\mathrm{T}}$ in ", the quality of the adjustment to GSC 1.2 used to determine the significant p.m. lower limit $\sigma_{\mu}$ in $0.01^{\prime \prime} \mathrm{yr}^{-1}$, the number $N$ of stars inventoried by APM in 2002, the number $n$ of stars inventoried visually in 1920, and the significant level in $B$-magnitude variability $\sigma_{\mathrm{m}}$ between exposures of the $N$ stars on a plate. Each plate is identified by a number indexing a file available on-line. The rms error $\sigma_{\mathrm{G}} \approx \frac{1}{3} \sigma_{\mu}$ is a one-dimensional test to qualify the adjustment for each of the astrographic plates relatively to GSC 1.2 and was found to verify $0.002 \leq \sigma_{\mathrm{G}} \leq 0.006^{\prime \prime} \mathrm{yr}^{-1}$, quite similar to the precision of other photographic p.m. surveys spanning 40 years (Hanson et al. 2004; Spagna et al. 1996). Stars with p.m. $\mu \geq$ $0.015^{\prime \prime} \mathrm{yr}^{-1}$, ten times smaller than the value commonly used (Lépine et al. 2002, 2003) and about three to four times larger than the mean p.m. error, are defined as high p.m. candidates.

It should be noted that the inventory ( $N$ stars per plate) did not identify as many stars as the number of targets measured in the 1920's ( $n$ stars); it was estimated that about one magnitude has been lost due to ageing of these short-exposure plates over more than a century. The geometric adjustment $\sigma_{\mathrm{T}}$ to the Tycho-2 catalog on the order of $0.3^{\prime \prime}$ suggests an image accuracy on the order of 5 microns comparable to the estimated errors $\Delta x$, $\Delta y$, but the main concern is the deterioration of plate contrast due to ageing. In order to check this result on the deep plates of the astrographic survey, the inventory of stars detected with APM on a set of 187 deep AC plates of the Northern Hemisphere is 
Table 1. Excerpt of the list of 104 short exposure Sydney observatory astrographic plates along the galactic equator: $(\alpha, \delta)=$ B1900 equatorial coordinates of plate center, $\sigma_{\mathrm{T}}=\mathrm{rms}$ error in " of the astrometric calibration to the Tycho- 2 catalogue at the epoch of the observations, $(N, n)=$ numbers of stars inventoried in 2002 and around $1920 \pm 10, \sigma_{\mu}=$ significant p.m. in $0.01^{\prime \prime} \mathrm{yr}^{-1}$ for $N$ stars matched with GSC 1.2, $\sigma_{\mathrm{m}}=$ significant magnitude variability between exposures on the plate for $N$ stars.

\begin{tabular}{ccccccccc}
\hline \hline$\alpha$ & $\delta$ & $\sigma_{\mathrm{T}}$ & Epoch & $N$ & $n$ & $\sigma_{\mu}$ & $\sigma_{\mathrm{m}}$ & id. \\
\hline $16^{\mathrm{h}} 36^{\mathrm{m}}$ & $-52^{\circ}$ & 0.26 & 1894 May 2 & 792 & 546 & 2.07 & 0.61 & 1436 \\
$09^{\mathrm{h}} 56^{\mathrm{m}}$ & $-53^{\circ}$ & 0.28 & 1900 Feb. 6 & 701 & 1088 & 1.37 & 0.45 & $136 \mathrm{rh}$ \\
$09^{\mathrm{h}} 18^{\mathrm{m}}$ & $-53^{\circ}$ & 0.28 & 1900 Jan. 26 & 333 & & 0.64 & 0.22 & $108 \mathrm{rh}$ \\
$09^{\mathrm{h}} 30^{\mathrm{m}}$ & $-53^{\circ}$ & 0.30 & 1899 March 14 & 798 & & 0.86 & 0.30 & $\mathrm{~d} 38992$ \\
$10^{\mathrm{h}} 18^{\mathrm{m}}$ & $-53^{\circ}$ & 0.32 & 1900 Feb. 6 & 548 & 716 & 0.98 & 0.48 & $141 \mathrm{rh}$ \\
$\ldots$ &... &.. &.. &.. &.. &.. &.. & $\ldots$. \\
\hline
\end{tabular}

Table 2. Excerpt of the list of 187 miscellaneous photographic plates of the Northern Hemisphere with a field of view of $2^{\circ} \times 2^{\circ}$ at a scale of $1^{\prime} \mathrm{mm}^{-1}$ (Vatican $\delta \in\left[55^{\circ}, 57^{\circ}\right]$, Uccle $\delta \in\left[33^{\circ}, 39^{\circ}\right]$, Paris $\delta \in\left[18^{\circ}, 24^{\circ}\right]$ and Bordeaux $\left.\delta \in\left[12^{\circ}, 16^{\circ}\right]\right):(\alpha, \delta)=\mathrm{B} 1900$ equatorial coordinates of the plate center at the epoch of observations, $(N, n)=$ numbers of stars inventoried in 2002 and around $1920 \pm 10$ : in most cases $N \ll n$, proving a degradation of plate contrast at present times.

\begin{tabular}{cccccc}
\hline \hline$\alpha$ & $\delta$ & Epoch & $N$ & $n$ & id. \\
\hline $18^{\mathrm{h}} 48^{\mathrm{m}}$ & $12^{\circ}$ & 1909 July 14 & 1951 & 2819 & $\mathrm{C} 12142$ \\
$18^{\mathrm{h}} 56^{\mathrm{m}}$ & $12^{\circ}$ & 1909 July 18 & 1343 & 1813 & $\mathrm{C} 12143$ \\
$19^{\mathrm{h}} 04^{\mathrm{m}}$ & $12^{\circ}$ & 1909 July 17 & 1219 & 1737 & $\mathrm{C} 12144$ \\
$19^{\mathrm{h}} 12^{\mathrm{m}}$ & $12^{\circ}$ & 1909 July 20 & 590 & 697 & $\mathrm{C} 12145$ \\
$19^{\mathrm{h}} 20^{\mathrm{m}}$ & $12^{\circ}$ & 1909 Aug. 19 & 234 & 819 & $\mathrm{C} 12146$ \\
.. & &.. & $\ldots$ & $\ldots$ &.. \\
\hline
\end{tabular}

given in Table 2. The set of $10^{4}$ deep AC photographic plates of the all-sky survey was never measured: the set was intended to provide paper charts through an expensive technique of engraving a copper plate and printing the atlas. In Table $2, \mathrm{~N}$ is the number of stars inventoried by APM, and $n$ the number inventoried by visual count on the star atlas produced at the epoch of the photographic exposure. Complete information for the $\mathrm{N}$ stars detected on these plates $(\alpha, \delta, \Delta \alpha, \Delta \delta, x, y, \Delta x, \Delta y, m, \Delta m)$ is available on-line ${ }^{2}$. Again, $n \geq N$ and comparing $\log N$ to $\log n$ reveals a loss of approximately half-a-magnitude from ageing of the old plates down to $B=14.0 \pm 0.5$. In this paper, the Sydney Observatory Galactic Survey (SOGS) is presented as a set of 650 astrographic plates selected along the galactic meridian $l=$ $(330 \pm 5)^{\circ},-64^{\circ} \leq b \leq-7^{\circ}$ and along the galactic equator $272^{\circ} \leq$ $l \leq 341^{\circ},|b| \leq 7^{\circ}$, observed in the 52 to 64 southern declination zone allocated to Sydney Observatory. The set of 650 plates is just a short sample of the set of $2 \times 10^{4}$ similar plates archived at Macquarie University. The purpose of SOGS is to archive stellar coordinates as early as the end of the 19th century, even with low accuracies, and to provide a catalogue of high p.m. stars suitable for investigating Galaxy disk kinematics up to a distance of $1 \mathrm{kpc}$ from the Sun.

\section{Sydney observatory deep astrographic plates}

The APM was also used to measure a set of 535 deep AC photographic plates observed in the Bordeaux astrographic survey zone $\left(+11^{\circ}\right.$ to $\left.+18^{\circ}\right)$ taken between 1890 and 1920 (Rapaport et al. 2006). The quality of the adjustment of a plate relative to the Tycho-2 catalogue is indicated by a scatter $\sigma$ (in ") plotted in Fig. 2 as function of the right ascension for the set of 535 Bordeaux plates (open circles) and the set of 650 Sydney

\footnotetext{
${ }^{2}$ See ftp://cdsarc.u-strasbg.fr/incoming/fresneau/misc
}

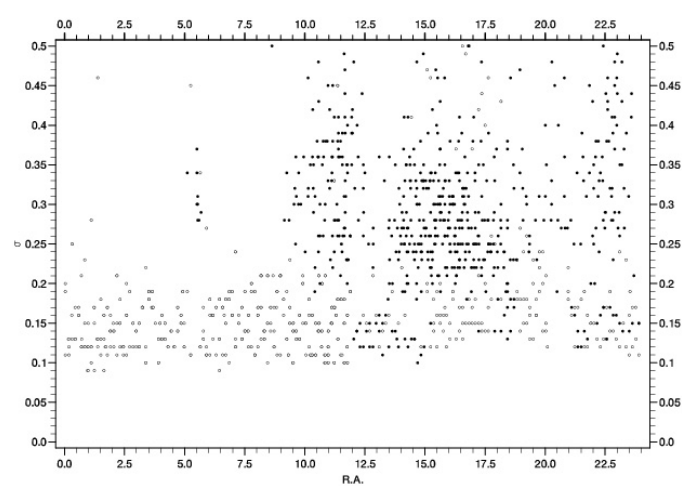

Fig. 2. The rms error $\sigma$ in " of the astrometric calibration relatively to the Tycho-2 catalogue of a set of 535 astrographic plates of the Bordeaux zone (open circles) and 650 plates of the Sydney zone (dots) is plotted as function of the Right Ascension (RA). A difference in quality is observed for the two sets of plates.

plates (dots). The Sydney plates have been selected along the galactic meridian $l=(330 \pm 5)^{\circ},-64^{\circ} \leq b \leq-7^{\circ}$ and along the galactic equator $272^{\circ} \leq l \leq 341^{\circ},|b| \leq 7^{\circ}$ and not all deep plates of the Sydney zone $-64^{\circ} \leq \delta \leq-52^{\circ}$ have been measured.

The scatter $\sigma$ of the Bordeaux astrographic plates is around $0.15^{\prime \prime}$ whilst that of the Sydney plates is around $0.35^{\prime \prime}$, corresponding respectively to accuracies of 2.5 and 5.5 microns in the measured $x, y$ coordinates. Since the photographic plate measurement is performed with the same machine, it is possible to demonstrate a difference in plate quality between the two observatories. In view of the propagation of the errors in the Tycho2 p.m. for stars in the Bordeaux declination zone $\left(+11^{\circ}\right.$ to $\left.+18^{\circ}\right)$ and in the Sydney zone $\left(-52^{\circ}\right.$ to $\left.-64^{\circ}\right)$, we estimate a general contribution on the order of $0.2^{\prime \prime}$ at the epoch of the AC observations (Høg et al. 2000), suggesting that the Bordeaux plates were measured by APM at the limit of a stellar image quality. Both Bordeaux and Sydney observatories being located at sea level, we did not investigate differential astronomic refraction effects over the $2^{\circ} \times 2^{\circ}$ field of view in each plate.

The inventory of stellar $x, y$ plate coordinates (in $\mathrm{mm}$ ) is provided on-line, along with magnitude $\mathrm{m}$ and equatorial positions $\alpha, \delta$ (equator and equinox 2000) for each star identified on the 258 individual deep photographic plates at the epoch of the observation of the Sydney Observatory Astrographic Program along the galactic meridian $l=330^{\circ} \pm 5^{\circ},-64^{\circ} \leq b \leq-7^{\circ}$. The metric $\Delta x, \Delta y$ (in microns) and radiometric $\Delta m$ errors, the estimated errors $\Delta \alpha, \Delta \delta$ (in ") on the equatorial stellar coordinates $\alpha, \delta$ are given for each star inventoried in the photographic plates. Table 3 identifies the plate center coordinates $\left(\alpha_{0}\right.$, $\delta_{0}$ ), the epoch of observations, the quality of the adjustment to Tycho-2 catalogue $\sigma_{\mathrm{T}}$ in ", the quality of the adjustment to 
Table 3. Excerpt of the list of 258 deep exposure astrographic plates selected from the Sydney Observatory Astrographic Program legacy along the galactic meridian $l=330^{\circ} \pm 5^{\circ},-64^{\circ} \leq b \leq 7^{\circ}:(\alpha, \delta)=\mathrm{B} 1900$ equatorial coordinates of plate center, $\sigma_{\mathrm{T}}=$ rms error in " of the astrometric calibration to the Tycho-2 catalogue at the Epoch of observations, $N=$ number of stars inventoried in $2002, \sigma_{\mu}=$ significant p.m. in $0.01^{\prime \prime} \mathrm{yr}^{-1}$ for stars matched with GSC1.2, $\sigma_{\mathrm{m}}=$ significant magnitude variability between exposures on a plate.

\begin{tabular}{cccccccc}
\hline \hline$\alpha$ & $\delta$ & $\sigma_{\mathrm{T}}$ & Epoch & $N$ & $\sigma_{\mu}$ & $\sigma_{m}$ & id. \\
\hline $05^{\mathrm{h}} 30^{\mathrm{m}}$ & $-05^{\circ}$ & 0.30 & 1895 Febr. 26 & 11968 & 0.92 & 0.0 & $\mathrm{~S} 2310$ \\
$23^{\mathrm{h}} 12^{\mathrm{m}}$ & $-52^{\circ}$ & 0.27 & 1927 Aug. 31 & 670 & 2.12 & 0.26 & $\mathrm{C} 1700$ \\
$23^{\mathrm{h}} 24^{\mathrm{m}}$ & $-52^{\circ}$ & 0.27 & 1927 Nov. 11 & 434 & 1.19 & 0.14 & $\mathrm{C} 1724$ \\
$23^{\mathrm{h}} 36^{\mathrm{m}}$ & $-52^{\circ}$ & 0.21 & 1927 Oct. 15 & 759 & 0.60 & 0.15 & $\mathrm{C} 1711$ \\
$15^{\mathrm{h}} 06^{\mathrm{m}}$ & $-53^{\circ}$ & 0.29 & 1900 May 1 & 8255 & 1.59 & 0.0 & S354rh \\
.. &.. &.. &... &.. &.. &.. &.. \\
\hline
\end{tabular}

GSC 1.2 used to determine the significant p.m. lower limit $\sigma_{\mu}$ in $0.01^{\prime \prime} \mathrm{yr}^{-1}$, the number $N$ of stars inventoried, and the rms error in $B$-magnitude $\sigma_{\mathrm{m}}$ between exposures on a plate identified by a plate number indexing a file available on-line ${ }^{3}$ for the deep plates along $l=\left(330^{\circ} \pm 5^{\circ}\right),-64^{\circ} \leq b \leq-7^{\circ}$ that were measured for this study. The same information is available in Table 3 of (Fresneau et al. 2005) for the 291 deep Sydney Observatory Astrographic Plates along the galactic equator $272^{\circ} \leq l \leq 341^{\circ}$, $|b| \leq 7^{\circ}$ and available on-line ${ }^{4}$.

We suggest that the information coming out from the Astrographic Program heritage is still of some use to derive stellar p.m. components with modern epoch observations (Ducourant et al. 2006; Sanner et al. 2001) and deserves to be archived.

\section{Catalogue of high p.m. stars}

The set of 650 deep AC plates in the 13 declination zones $\left(-64^{\circ} \leq \delta \leq-52^{\circ}\right)$ of the Sydney Observatory Astrographic Program along the galactic equator $272^{\circ} \leq l \leq 341^{\circ},|b| \leq 7^{\circ}$, and the galactic meridian $l=(330 \pm 5)^{\circ},-64^{\circ} \leq b \leq-7^{\circ}$ of the Southern Milky Way (a set of about $1.9 \times 10^{6}$ stars) has been matched with the GSC 1.2: this process provides equatorial coordinates and $V$-magnitude from the GSC 1.2, $B$-magnitude and significant p.m. components $\left(\mu \geq \sigma_{\mu}\right)$ for about $4.8 \times 10^{5}$ stars, according to the reduction technique described in Sect. 1 .

Figure 3 plots the distribution of the number $N$ of total p.m. $\mu$ for the set of $4.8 \times 10^{5}$ stars. The main result shown in Fig. 3 is to highlight a two-peak distribution with an excess of stars around $\mu=(0.2 \pm 0.1)^{\prime \prime} \mathrm{yr}^{-1}$ when the significant level for the p.m. distribution is about $(0.015 \pm 0.005)^{\prime \prime} \mathrm{yr}^{-1}$. This twopeak distribution is interpreted as the bias in a magnitude-limited and distance-limited stellar sample. If we assume that high velocity stars around the Sun qualify when $V \geq 62 \mathrm{~km} \mathrm{~s}^{-1}$, then the p.m. limit of $\mu \approx 0.1^{\prime \prime} \mathrm{yr}^{-1}$ corresponds to a distance $D=$ $\frac{V}{4.74 \mu} \approx 130 \mathrm{pc}$. Since the stellar sample is limited up to apparent $V$-magnitude $\approx 13$ and $B$-magnitude $\approx 14.5$, then the absolute $V$ magnitude limit at $130 \mathrm{pc}$ is $\approx 7.5$. The overdensity of stars with $\mu=(0.200 \pm 0.125)^{\prime \prime} \mathrm{yr}^{-1}$ is the excess of stars fainter than absolute $V$-magnitude 7.5 and of color $B-V \leq 1.5$, corresponding to the M0-M2 dwarfs contribution in the stellar sample. An absolute $V$-magnitude fainter than 6 is also the brightest limit for halo star detection (Bahcall \& Soneira 1980). The overdensity around $\mu=0.2^{\prime \prime} \mathrm{yr}^{-1}$ is the contribution of faint disk and halo dwarf candidates in the distance-limited stellar sample by the p.m. cutoff primitive selection.

\footnotetext{
${ }^{3}$ See

ftp://cdsarc.u-strasbg.fr/incoming/fresneau/merid ${ }^{4}$ See

ftp://cdsarc.u-strasbg.fr/incoming/fresneau/sydney
}

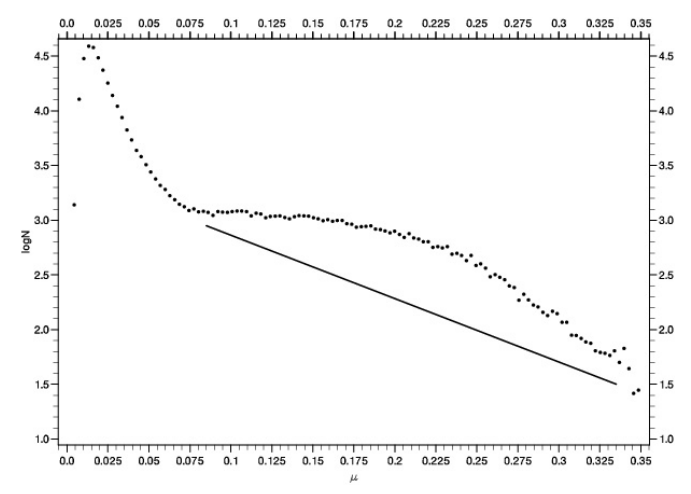

Fig. 3. Distribution of the number of stars $N$, with significant total p.m. component $\mu$ in " $\mathrm{yr}^{-1}$ matched between a set of 650 deep Sydney Observatory AC Plates and the GSC I, version 1.2. The contribution of dwarfs fainter than absolute magnitude 7.5 and closer than $130 \mathrm{pc}$ is observed in excess over the thick line when $0.075 \leq \mu \leq 0.3^{\prime \prime} \mathrm{yr}^{-1}$ in our magnitude-limited stellar sample.

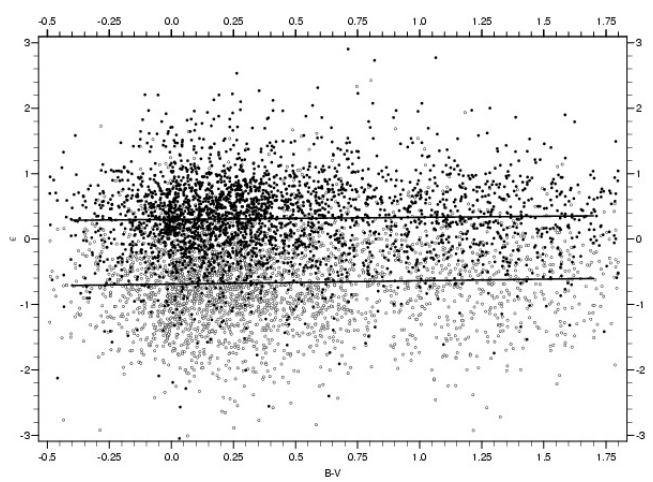

Fig. 4. Differences $\varepsilon=\Delta \mu_{l}$ (open circles) or $\Delta \mu_{b}$ (dots) in $0.001^{\prime \prime} \mathrm{yr}^{-1}$ between the Tycho-2 p.m. components and our p.m. determination for stars in the region of $\eta$ Carinae. The thick lines represent the linear fit of $\varepsilon$ with color $B-V$, suggesting systematic errors well below the estimated p.m. mean errors $\simeq 0.002^{\prime \prime} \mathrm{yr}^{-1}$.

The difference (in $10^{-3 \prime \prime} \mathrm{yr}^{-1}$ ) between the Tycho 2 space-borne p.m. and our ground-based p.m. determinations in the Carina region $\alpha \in\left[10^{\mathrm{h}}, 11^{\mathrm{h}}\right], \delta \in\left[-63^{\circ},-57^{\circ}\right]$ (Fresneau et al. 2003), are examined and Fig. 4 shows the difference $\varepsilon$ in $10^{-3 \prime \prime} \mathrm{yr}^{-1}$ of $\Delta \mu_{l}$ (open circles) and $\Delta \mu_{b}$ (dots). In Fig. 4 we rediscover the excess in numbers of Tycho 2 stars around $B-V=0.6$ and around $B-V=1.2$ (Perryman et al. 1997 Fig. 3.5.24). There appears to be no systematic trend with colour larger than $0.5 \times 10^{-3 \prime \prime} \mathrm{yr}^{-1}$ per magnitude over the range $0 \leq B-V \leq 1.2$ and we rediscover the scatter of $2.5 \times$ $10^{-3 \prime \prime} \mathrm{yr}^{-1}$ in Tycho-2 p.m. similar to the scatter $\sigma_{\mathrm{G}}$ estimated in our observations. A systematic offset $\varepsilon=-0.8 \times 10^{-3 \prime \prime} \mathrm{yr}^{-1}$ is 
estimated for $\Delta \mu_{l}$ and $\varepsilon=0.2 \times 10^{-3 \prime \prime} \mathrm{yr}^{-1}$ for $\Delta \mu_{b}$ between the Tycho- 2 p.m. and our observations. With the observed dispersion of $\pm 2 \times 10^{-3 \prime \prime} \mathrm{yr}^{-1}$, we can assume that up to a distance of $500 \mathrm{pc}$ from the Sun, $(V, W)$ velocity components can be derived with rms error $\approx 5 \mathrm{~km} \mathrm{~s}^{-1}$. It should be emphasized that our groundbased observations are an extension of the Tycho 2 catalogue to fainter targets. The same information $\left(\Delta \mu_{l}, \Delta \mu_{b}\right)$ was analyzed for stars matching Tycho-2 and the deep plates around Southern Coalsack $\alpha \in\left[12^{\mathrm{h}}, 14^{\mathrm{h}}\right], \delta \in\left[-65^{\circ},-57^{\circ}\right]$, Southern Centaurus $\alpha \in\left[11^{\mathrm{h}}, 12^{\mathrm{h}}\right], \delta \in\left[-64^{\circ},-58^{\circ}\right]$, and LMC $\alpha \in\left[5^{\mathrm{h}}, 6^{\mathrm{h}}\right], \delta \in$ $\left[-71^{\circ},-65^{\circ}\right]$. Again, the difference in p.m. components between our measurements and the Tycho- 2 data show a systematic offset on the order of $\varepsilon \approx-0.8 \times 10^{-3 \prime \prime} \mathrm{yr}^{-1}$ in $\Delta \mu_{l}$ and $\varepsilon \approx \pm 0.2 \times$ $10^{-3 \prime \prime} \mathrm{yr}^{-1}$ in $\Delta \mu_{b}$. These results suggest an overall accuracy on the order of $\pm 2 \times 10^{-3 \prime \prime} \mathrm{yr}^{-1}$. In our study, we did not investigate the systematic offsets $|\varepsilon| \leq 10^{-3 \prime \prime} \mathrm{yr}^{-1}$, since we consider high p.m. stellar candidates when $\mu \geq(0.015 \pm 0.005)^{\prime \prime} \mathrm{yr}^{-1}$.

\section{Empirical calibration of distances from total p.m.}

The Reduced Proper Motion $H=B+5+5 \log \mu$ with B the apparent magnitude and $\mu$ the total p.m. component in " $\mathrm{yr}^{-1}$, is a convenient tool to segregate giants from dwarfs in a p.m. survey. In a previous study (Fresneau et al. 2003), based on the Tycho-2 catalogue, the following relation was obtained to estimate the mean absolute magnitude of the stellar sample over the celestial sphere: $\bar{M}_{B}=(-3.76 \pm 0.01)+(0.711 \pm 0.001) H$. This relation describes the mixing of stellar ages among the Main Sequence B5 to G2 stars detected around the mean transverse velocity $V_{\mathrm{t}} \approx 45 \mathrm{~km} \mathrm{~s}^{-1}$ in the solar vicinity. We emphasize that the mean relation $\bar{M}_{B} \approx H-5 \log V_{\mathrm{t}}\left(V_{\mathrm{t}}\right.$ in $\left.\mathrm{AU} \mathrm{yr}^{-1}\right)$ or $\bar{M}_{B}=H-4.89$ is the exact relation that should be used with $V_{\mathrm{t}}=45 \mathrm{~km} \mathrm{~s}^{-1}$. The error resulting between the adopted calibration and the theoretical one is $\Delta M_{B} \approx 0.3 M_{B}-1.13$ or about $1 \mathrm{mag}$. We are aware of the low accuracies, but the analysis of a p.m. survey, in the framework of Pop. I and II color-magnitude sequences, can separate kinematically homogeneous stellar systems and identify genuine stellar candidates for further observations (Fresneau 1990; Cuisinier et al. 1994; Salim \& Gould 2002).

In the present study, the same relation $\bar{M}_{B}=-3.76+0.711 H$ is applied to estimate the absolute magnitude of $4.8 \times 10^{5}$ stars down to $B=14.5$ with $\mu \geq(0.015 \pm 0.005)^{\prime \prime} \mathrm{yr}^{-1}$. This relation is valid for stars in the solar neighborhood of mean tranverse velocity $V_{\mathrm{t}}=45 \mathrm{~km} \mathrm{~s}^{-1}$ and is also similar to the relation established when using different stellar samples (Jones 1972).

The matching process of SOGS $\cap$ GSC1.2 along the galactic meridian $l=(330 \pm 5)^{\circ},-64^{\circ} \leq b \leq-7^{\circ}$ and the galactic equator $272^{\circ} \leq l \leq 341^{\circ},|b| \leq 7^{\circ}$ provides significant total p.m. $\mu$ for a subset of $4.8 \times 10^{5}$ stars with $\mu \geq(0.015 \pm 0.005)^{\prime \prime} \mathrm{yr}^{-1}$. The $V, W$ velocity components are computed ( $V$ in the direction of galactic rotation, $W$ perpendicular to the galactic plane), directly from the observations $\left(\mu_{l}, \mu_{b}, l, b\right.$, with no radial velocity information); $\frac{V}{k D}=\mu_{b} \sin b \sin l+\mu_{l} \cos b \cos l$ and $\frac{W}{k D}=\mu_{b} \cos b$ where $\mathrm{D}$ is expressed in pc, the galactic components $\mu_{l}, \mu_{b}$ of the total p.m. $\mu$ in " $\mathrm{yr}^{-1}$, the conversion factor $k=4.74$, and $V, W$ in $\mathrm{km} \mathrm{s}^{-1}$ (Johnson \& Soderblom 1987). Stellar distance D is derived from a statistical absolute magnitude $M_{B}=-3.76+$ $0.711 H_{B}$, where $H_{B}=m_{B}+5+5 \log \mu\left(m_{B}\right.$ is the star apparent $B$-magnitude and $\mu$ the total p.m. component in " $\left.\mathrm{yr}^{-1}\right)$. The main uncertainty in these results is in the derivation of $D$ since the absolute magnitude can be in error of up to one magnitude (corresponding to a relative accuracy of $\sim 50 \%$ in $D$ ). The relation established (Binney et al. 2000) for the transverse velocity

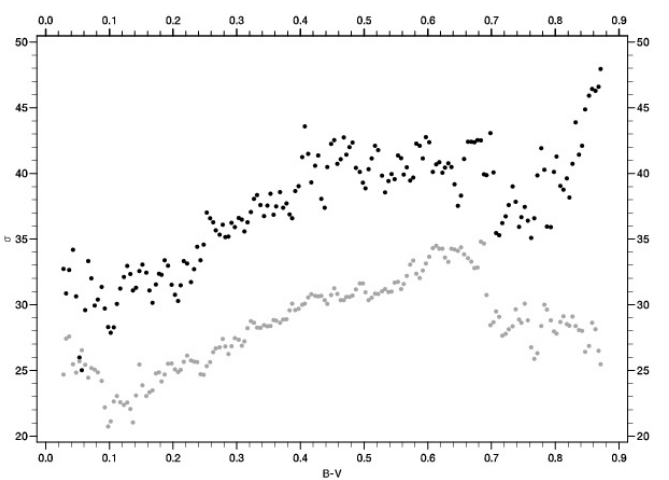

Fig. 5. Averaged residual velocity dispersion $\sigma_{V}$ (black dots) and $\sigma_{W}$ (grey dots) in $\mathrm{km} \mathrm{s}^{-1}$ derived from a set of stars along the 4th galactic quadrant and up to a distance of $300 \mathrm{pc}$ from the Sun, as function of the colour index $B-V$. A steady increase of the transverse velocity is rediscovered as the result of the selection effect in our study. Estimated accuracies on $\sigma_{V}$ and $\sigma_{W}$ are $\simeq 3 \mathrm{~km} \mathrm{~s}^{-1}$.

$S$ of stars at a distance $D \leq 80$ pc from the Sun estimates $S \simeq$ $25 \mathrm{~km} \mathrm{~s}^{-1}$ for stars with $B-V \leq 0.2$ and $S \simeq 50 \mathrm{~km} \mathrm{~s}^{-1}$ for stars with $B-V \geq 0.9$. This relation is fundamental to justify our statistical parallax determination. We check the approximate relation $M_{B}=-3.76+0.711 H_{B}$ in the investigation a posteriori of the mean transverse velocity of $S \simeq 45 \mathrm{~km} \mathrm{~s}^{-1}$ for stars detected at a distance $D \leq 300 \mathrm{pc}$ from the Sun.

The absence of radial velocity information affects the $V$ component dispersion $\sigma_{V}$ as $(\sin l \cos b)^{2}$ and the $W$ component dispersion $\sigma_{W}$ as $(\sin b)^{2}$, below the estimated errors of $\sim 50 \%$ in our determination of stellar distance for F-G stellar candidates selected along the regions $|b| \leq 7^{\circ}$ and $l=(330 \pm 5)^{\circ}$. Once the $V, W$ velocity components are computed, the equation $f(V, W)=0$ of an ellipse is minimized using a stepwise regression method and the best linear unbiased estimation of the 5 unknowns of the velocity ellipse, $V_{0}, W_{0}, \sigma_{V}, \sigma_{W}, \rho$ is derived after a weighted least squares fit at $10 \%$ level of significance in the $\chi^{2}$-test (Branham 2004):

$$
\begin{aligned}
f(V, W)= & \left(\frac{V-V_{0}}{\sigma_{V}}\right)^{2}-2 \rho \frac{\left(V-V_{0}\right)\left(W-W_{0}\right)}{\sigma_{V} \sigma_{W}} \\
& +\left(\frac{W-W_{0}}{\sigma_{W}}\right)^{2}-1+\rho^{2}
\end{aligned}
$$

The tilt term of the velocity ellipse is $\theta=\frac{1}{2} \arctan \frac{2 \rho \sigma_{V} \sigma_{W}}{\sigma_{V}^{2}-\sigma_{W}{ }^{2}}$.

The data being used here is rudimentary: the photographic $B$ and $V$ magnitudes are accurate to $\pm 0.3 \mathrm{mag}$, and equatorial coordinates to $\pm 0.3^{\prime \prime}$ for a set of $1.9 \times 10^{6}$ stars of limiting magnitude $B=14.5 \pm 0.3$ at mean Epoch $1912 \pm 20$. For the later Quick- $V$ data (Lasker et al. 1990) the limiting magnitude is $V=$ $13.5 \pm 0.3$ at mean Epoch $1985 \pm 5$. Comparing these two photographic surveys observed some 80 years apart, it appears that $\mu=$ $(0.015 \pm 0.005)^{\prime \prime} \mathrm{yr}^{-1}$ is a significant value. Although this selection introduces a bias towards stars at a specific velocity (corresponding to $21 \pm 7 \mathrm{~km} \mathrm{~s}^{-1}$ at a distance of $300 \mathrm{pc}$ ), the colour index $B-V$ can be employed to derive an indication of stellar spectral type and stellar radius by segregating dwarfs from giants in a Reduced Proper Motion Diagram $H_{B}, B-V$ (Jones 1972), and halo from disk dwarfs as well (Evans 1992; Lépine et al. 2003).

Figure 5 shows the $\sigma_{V}$ and $\sigma_{W}$ velocity dispersions (in $\mathrm{km} \mathrm{s}^{-1}$ ) derived in the weighted least squares fit of $f(V, W)=0$ for the stellar system detected at a distance 
$D \leq 300 \mathrm{pc}$ and ordered by colour index $B-V$ in groups of 105 stars, (a weight of about $\sqrt{100}$ for the internal $5 \mathrm{~km} \mathrm{~s}^{-1}$ accuracy in the velocity dispersions $\sigma_{V}$ and $\sigma_{W}$.) The continuous increase of the known transverse velocity dispersion for stars in the solar neighborhood as observed by (Binney et al. 2000) suggests that F-G disk dwarfs are detected. The observed heating $\sigma$ in $\sigma_{V}$ between $B-V=0.2, \sigma_{V} \simeq 33 \mathrm{~km} \mathrm{~s}^{-1}$ and $B-V=0.9, \sigma_{V} \simeq$ $41 \mathrm{~km} \mathrm{~s}^{-1}$ can be found in $\mathrm{km} \mathrm{s}^{-1}$ by assuming no correlation in the increase of $\sigma_{V}$, such that $33^{2}+\sigma^{2}=41^{2}$. A value of $\sigma \simeq$ $25 \mathrm{~km} \mathrm{~s}^{-1}$ is actually observed, linked to the age-velocity dispersion over $\simeq 5$ Gyr (Binney et al. 2000; Edvardsson et al. 1993). These results give some confidence in the analysis of the sample of $4.8 \times 10^{5}$ stars with the photometric and astrometric errors given above. Figure 5 deals with averaged values of 105 stars per data point through a weighted least squares fit that suggests an internal dispersion error of $5 \mathrm{~km} \mathrm{~s}^{-1}$, and so, in spite of many uncertainties, it seems interesting to explore the stellar system classified from this survey with a well-known bias (apparent magnitude $m_{B} \leq 14.5, \mu \geq 0.015 \pm 0.005^{\prime \prime} \mathrm{yr}^{-1}$ ).

\section{Selection effects and kinematical bias}

Figure 6 shows the averaged value of the scatters (in $\mathrm{km} \mathrm{s}^{-1}$ ) $\sigma_{V}$, $\sigma_{W}$ in the $V$ and $W$ components of the stellar residual velocities computed for groups of 105 stars ordered by galactic height $z$ (in pc) out of the same subset of stars detected at a distance $D \leq$ $300 \mathrm{pc}$, as function of the height $z$ over the galactic plane. These dispersions $\sigma_{V}$ and $\sigma_{W}$ are computed as the best linear unbiased estimators after a least squares fit of the equation of an ellipse $f(V, W)=0$ assumed for the distribution of the $(V, W)$ velocity components of 105 stars ordered by height $z$, with $f(V, W)=$ $\left(\frac{V-V_{0}}{\sigma_{V}}\right)^{2}-2 \rho \frac{\left(V-V_{0}\right)\left(W-W_{0}\right)}{\sigma_{V} \sigma_{W}}+\left(\frac{W-W_{0}}{\sigma_{W}}\right)^{2}-1+\rho^{2}$. There is a conspicuous effect in the $\sigma_{V}$ dispersion component over the interval $|z| \leq 25 \mathrm{pc}$ and also for stars outside this range of $z$, with $\frac{\sigma_{W}}{\sigma_{V}} \sim$ 0.75 close to the equilibrium ratio estimated for isotropic velocity distribution (Jenkins \& Binney 1990). The sample of stars along the galactic equator $272^{\circ} \leq l \leq 341^{\circ},|b| \leq 7^{\circ}$ affects the galactic height sample $|z| \leq 50 \mathrm{pc}$; the absence of radial velocity information infers a bias in the $\sigma_{V}$ and $\sigma_{W}$ velocity dispersions as respectively $(\sin l \cos b)^{2}$ and $(\sin b)^{2}$. Along the galactic equator $272^{\circ} \leq l \leq 341^{\circ}$, the effect in $\sigma_{V}$ is on the order of $30 \%$, whilst along meridian $330^{\circ}$, between $-64^{\circ} \leq b \leq 7^{\circ}$ the effect in $\sigma_{W}$ is also about $30 \%$ but dispersions in $\sigma_{V}$ and $\sigma_{W}$ are higher when $z \leq-50 \mathrm{pc}$. Additionally, $\sigma_{V} \simeq 38 \mathrm{~km} \mathrm{~s}^{-1}$ when $-50 \leq z \leq$ $-20 \mathrm{pc}$ and $\sigma_{V} \simeq 34 \mathrm{~km} \mathrm{~s}^{-1}$ when $20 \leq z \leq 50 \mathrm{pc}$. Based on the estimated accuracy of $5 \mathrm{~km} \mathrm{~s}^{-1}$ derived above, the observed heating $\sigma \mathrm{km} \mathrm{s}^{-1}$ in $\sigma_{V}$ if $|z| \leq 25$ pc can be investigated between $\sigma_{V}=35 \mathrm{~km} \mathrm{~s}^{-1}$ and $\sigma_{V}=48 \mathrm{~km} \mathrm{~s}^{-1}$ which is observed at $z=0$. It is assumed that $\sigma$ is uncorrelated with the background level of $\sigma_{V}=35 \mathrm{~km} \mathrm{~s}^{-1}$ and so $\sigma^{2}+35^{2}=48^{2}$ leads to $\sigma=35 \mathrm{~km} \mathrm{~s}^{-1}$ at $z=0$. Since $\sigma_{W}$ appears to be constant with $z$, then the stellar system selected from our observations is well-mixed over the interval $-80 \leq z \leq 50 \mathrm{pc}$, and $\frac{\partial \sigma}{\partial z} \simeq 1.5 \mathrm{~km} \mathrm{~s}^{-1} \mathrm{pc}^{-1}$ for $|z| \leq 25 \mathrm{pc}$.

What is the the heating mechanism that contributes to $\sigma=$ $35 \mathrm{~km} \mathrm{~s}^{-1}$ ? With the rudimentary data we deal with, we do not intend to identify the relative importance of the two heating mechanisms of cloud scattering and spiral wave heating (Sellwood \& Binney 2002). Since the anisotropy $\frac{\sigma_{V}}{\sigma_{W}}$ is observed in the stellar sample at $|z| \leq 25 \mathrm{pc}$, it is better to ask what is the interaction between the stellar systems that are associated with a 25 pc half-thickness of the Galaxy disk with the gaseous disk? We know that $\frac{\sigma_{V}}{\sigma_{R}}$ in the disk is increased by cloud scattering and reduced by wave heating (Jenkins \& Binney 1990).

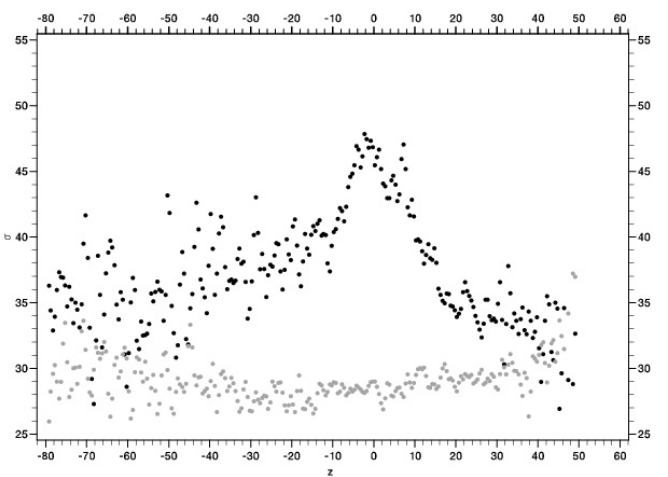

Fig. 6. Averaged residual velocity dispersion $\sigma_{V}$ (black dots) and $\sigma_{W}$ (grey dots) in $\mathrm{km} \mathrm{s}^{-1}$ derived from a set of stars along the fourth galactic quadrant and up to a distance of $300 \mathrm{pc}$, as function of the height $z$ in pc over the galactic plane. A $35 \mathrm{~km} \mathrm{~s}^{-1}$ heating at $z=0$ is observed over a thickness of $25 \mathrm{pc}$ around the Galaxy midplane.

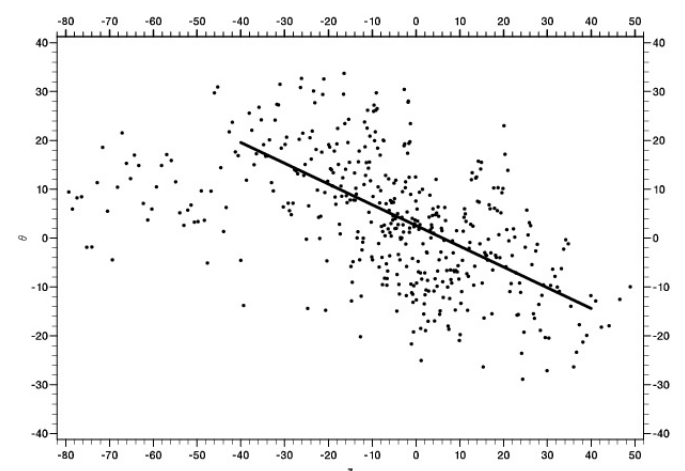

Fig. 7. The averaged tilt $\theta$ in degrees of the stellar velocity ellipsoid projected onto the $(V, W)$-plane is interpreted as a velocity anisotropy leading to a spin of $\frac{\partial \theta}{\partial z}=\left(\frac{1}{2} \pm \frac{1}{4}\right)^{\circ} \mathrm{pc}^{-1}$ in the direction of the galactic differential rotation as function of the galactic height $z$ in pc.

A stellar disk is heated as $\frac{\Delta V}{V} \simeq \frac{1}{2} \frac{\Delta \Sigma}{\Sigma}$ where $\Delta V$ is the increase in velocity scatter and $\Delta \Sigma$ the increase in surface density. In the solar neighborhood, $\Sigma \sim 50 M_{\odot} \mathrm{pc}^{-2}$ (Holmberg \& Flynn 2000; Bienaymé et al. 2006) and $V \sim 235 \mathrm{~km} \mathrm{~s}^{-1}$ are the averaged values of the disk surface mass density and the disk velocity. We assume that we detect velocity scattering from the potential fluctuation $\Delta \Sigma$ in the disk of surface density $\Sigma$ by giant molecular clouds (GMC) and by bits of transient spiral structure in the area covered by $|z| \leq 25$ pc. Figure 6 suggests $\frac{\Delta V}{V}=\frac{1}{2} \frac{\Delta \Sigma}{\Sigma} \simeq 0.1$ with the set of stars at a height of $\pm 25 \mathrm{pc}$ and at a distance $D \leq 300 \mathrm{pc}$ out of the sample of $4.8 \times 10^{5}$ stars selected from SOGS. The known value of $\Delta \Sigma \simeq 10 M_{\odot} \mathrm{pc}^{-2}$ is rediscovered for the total surface mass density of atomic and molecular gas in the solar neighborhood from our observation of $\frac{1}{2} \frac{\Delta \Sigma}{\Sigma}=0.1$.

By analogy with the cool disk models (Shu 1969; Binney 2005), $\frac{\sigma_{V}}{\sigma_{W}}$ can be considered as an anisotropy parameter. The tilt term of the velocity ellipse is $\theta=\frac{1}{2} \arctan \frac{2 \rho \sigma_{V} \sigma_{W}}{\sigma_{V}^{2}-\sigma_{W}{ }^{2}}$ and can be used as a means of controlling the effect of disk surface density variation. This tilt term is more effective in tracing the deflection of a stellar system in velocity space than the scattering $\Delta V$ of the stellar system by a mass $M$ encountered at speed $V$ and impact parameter $b$ and where $\Delta V \propto \frac{M}{b V}$ (Fresneau et al. 2003).

Figure 7 shows the averaged value of the tilt term $\theta$ (in degrees) derived from the $\sigma_{V}, \sigma_{W}$ and $\rho$ quantities estimated in the $(V, W)$-plane for the successive samples of 105 stars selected earlier. A linear variation of $\theta$ with the height $z$ (in pc) above the 
galactic plane is observed when $|z| \geq 25$ pc and $\theta \simeq 0$ when $z \leq$ $-25 \mathrm{pc}$ or $z \geq 25 \mathrm{pc}$. The tilt term of the velocity ellipse is surprisingly high $\left(|\theta| \simeq 20^{\circ}\right)$ at the interface of \pm 25 pc suggesting a kinematical effect due to the viscosity between two different layers of mean surface mass density $\Sigma \pm \Delta \Sigma$ over the galactic plane (Bertin \& Cava 2006). In the observations, the increase in the velocity scatter $\sigma_{V}$ can be seen in the direction of the Galaxy rotation and in the anisotropy angle $\theta$ over the $50 \mathrm{pc}$ thickness with a constant scatter $\sigma_{W}$. Note that the entire sample of stars detected at a height lower than $25 \mathrm{pc}$ and at a distance up to $300 \mathrm{pc}$ is affected and that the tilt $\theta$ is sensitive when stars cross the interface at the height of $\pm 25 \mathrm{pc}$. Considering the galactic disk as a two-fluid system, it is possible to identify the contribution of the gas+dust $(\Delta \Sigma)$ to the mean surface mass density of the thin disk $(\Sigma)$ in the observed tilt term $\theta$.

The main result of the present section is to show that the straightforward analysis of the distribution of p.m. in a magnitude-limited sample survey can be used to extract a distance-limited sample of stars of $B-V \simeq 0.62 \pm 0.30$ with a controlled kinematical bias. Foreground stars are segregated from background stars when the p.m. component is larger than $(0.015 \pm 0.005)^{\prime \prime} \mathrm{yr}^{-1}$ in the present survey down to apparent $B$-magnitude $14.5 \pm 0.5$.

\section{Selection of disk crossing stars}

The increase of the stellar velocity dispersion $\sigma_{V}$ in the direction of the galactic rotation is observed over the galaxy disk thickness of $50 \mathrm{pc}$. The velocity ellipse adjusted in the $(V, W)$-plane is considered as the projection of the velocity ellipsoid and there is a tilt term $\theta$ over the galactic plane varying with height $z$ when $|z| \leq 25 \mathrm{pc}$ : the ellipsoid is apparently spinning at a rate $\frac{\partial \theta}{\partial z} \simeq$ $\frac{1}{2}{ }^{\circ} \mathrm{pc}^{-1}$ around its long-axis.

For each star in the sample of $4.8 \times 10^{5}$ stars, a height $z$ and a $(V, W)$ velocity can be computed from the observations $\left(\mu_{b}\right.$, $\left.\mu_{l}, D, l, b\right)$. We derive $\theta \propto z$ (with $\frac{\partial \theta}{\partial z} \simeq \frac{1}{2}^{\circ} \mathrm{pc}^{-1}$ ) and $\rho=$ $\frac{1}{2} \tan 2 \theta \frac{\sigma_{V}^{2}-\sigma_{W}{ }^{2}}{\sigma_{V} \sigma_{W}}$ is computed with $\sigma_{V} \simeq 35 \mathrm{~km} \mathrm{~s}^{-1}$ and $\sigma_{W} \simeq$ $28 \mathrm{~km} \mathrm{~s}^{-1}$. The heating effect in $\sigma_{V}$ over the $25 \mathrm{pc}$ half thickness shown in Fig. 6 is correlated with the $\frac{\partial \theta}{\partial z}=\frac{1}{2}{ }^{\circ} \mathrm{pc}^{-1}$ anomaly. We compute $f(V, W)={\frac{V}{\sigma_{V}}}^{2}-2 \rho \frac{V}{\sigma_{V}} \frac{W}{\sigma_{W}}+{\frac{W}{\sigma_{W}}}^{2}-1+\rho^{2}$ and we estimate a probability for a star to belong to the ellipse $f(V, W)=0$ in using a $\chi^{2}$ test.

In this formulation, $V_{0}=W_{0}=0$ is assumed for the subset of stars, since the tilt term $\theta$ is considered to be the main anisotropy parameter of the velocity ellipse. This is just an approximation but the observed relation $\theta \propto z$ is used to select the subset from the original sample with a controlled bias in the $(V, W)$ velocity space. There are no theoretical arguments to justify the simple linear relation between $\theta$ and $z$, but the results of this selection provides a way to estimate the probability for a star to trace the observed spin rate of the velocity ellipse over the finite disk thickness.

In this work, the main observational uncertainty remains the distance determination since the absolute magnitude $M_{B}$ is based on the the reduced proper motion $H_{B}=m_{B}+5+5 \log \mu$ which deals with the observed quantities (apparent magnitude $m_{B}$ and total p.m. component $\mu$ ). The determination of the distance is completely dependent of the p.m. and the result of SOGS is to approximate for each of the $4.8 \times 10^{5}$ stars a mean parallax $\pi$ and its error $\Delta \pi$ resulting from the errors in total p.m. $\Delta \mu \simeq$ $0.003^{\prime \prime} \mathrm{yr}^{-1}$ and in apparent magnitude $\Delta m_{B} \simeq 0.3$. Since we observe, through the spin rate of the velocity ellipsoid, a stellar

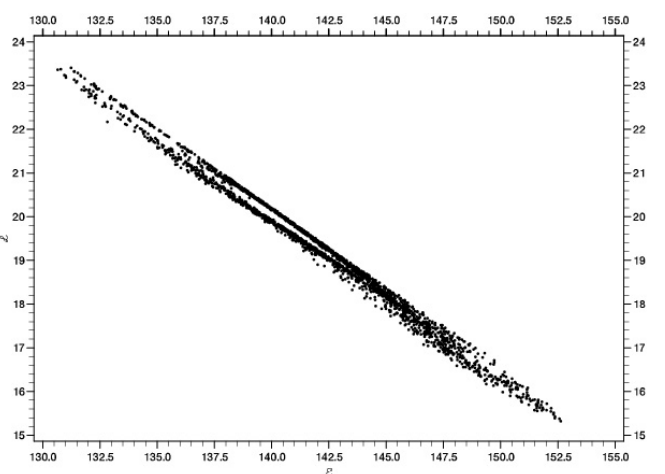

Fig. 8. Lindblad diagram $\left(E, L_{z}\right), \quad E$ in $10^{3}\left(\mathrm{~km} \mathrm{~s}^{-1}\right)^{2}, L_{z}$ in $10\left(\mathrm{kpc} \mathrm{km} \mathrm{s}^{-1}\right)$ for a set of stars at a height $|z| \leq 25 \mathrm{pc}$ over the galactic plane and at a distance $D \leq 300 \mathrm{pc}$ from the Sun, with $(V, W)$ velocity components distributed according to the tilt $\theta$ plotted in Fig. 7. The offset of $\delta E=\Omega_{p} \delta L_{z}$ is a transfer of angular momentum to energy for extreme disk crossing stars.

movement perpendicular to the galactic plane coupled with the azimuthal motion, we suggest a way to approach the statistical property of the general circulation at the interface between the extreme and thin disks of our Galaxy.

So as to derive the alignment of the velocity ellipsoid in a plane-parallel approximation of the Galaxy disk, this survey needs information on the third integral of motion that can be provided by the line-of-sight velocity available in other surveys (Steinmetz et al. 2006). In view of the results based on $(V, W)$ velocity components, it is suggested that the p.m. component perpendicular to the plane $\mu_{b}$ and the observed p.m. component in the plane $\mu_{l}$ show an anisotropy for stars along the galactic equator $\frac{\left\langle\mu_{l}\right\rangle}{\left\langle\mu_{b}\right\rangle} \propto \frac{\sigma_{V}}{\sigma_{W}} \sim 1.3$ that can be easily verified from the data available from SOGS, since we rediscover in $\frac{\sigma_{V}}{\sigma_{W}}$ the known ratio of the velocity ellipsoid dispersion axes of $\sigma_{U}: \sigma_{V}: \sigma_{W}=2.2: 1.4: 1$ (Binney \& Merrifield 1998) for stars of the solar neighborhood.

Figure 8 plots the Lindblad diagram $\left(L_{z}, E\right)$ (May \& Binney 1986 ) for the set of stars identified at a distance $D \leq 300 \mathrm{pc}$ and at a height over the galactic plane $z \leq 25 \mathrm{pc} . E$, in $10^{3}\left(\mathrm{~km} \mathrm{~s}^{-1}\right)^{2}$, is the sum of the kinetic and the binding energy per unit mass, and $L_{z}$, in $10\left(\mathrm{kpc} \mathrm{km} \mathrm{s}^{-1}\right)$, is the angular momentum around the $z$-axis per unit mass:

$L_{z}=R V$ where $R(\mathrm{kpc})$ is the distance to the galactic center and $V \mathrm{~km} \mathrm{~s}^{-1}$ the stellar velocity in the direction of the galactic rotation $\left(V=v+V_{\odot}\right.$ where $\left.V_{\odot}=235 \mathrm{~km} \mathrm{~s}^{-1}\right)$.

$E=\frac{1}{2}\left(u^{2}+V^{2}+w^{2}\right)^{\frac{1}{2}}-\Phi(x, y, z)$ where $(u, v, w) \mathrm{km} \mathrm{s}^{-1}$ the stellar velocity and $\Phi(x, y, z)$ the Plummer galactic potential (Carlberg \& Innanen 1987) at $(x, y, z)$ in the heliocentric Cartesian system ( $x$-axis in the direction of the galactic center, $y$ axis in the direction of the galactic rotation, $z$-axis perpendicular to the galactic plane).

Two conspicuous systems can be seen in Fig. 8 fitting the two straight lines corresponding to two different circular velocities in the galactic plane with lower and higher angular momentum around the $Z$-axis. The offset $\delta L_{z}$ of $\sim 1 \%$ along the $L_{z}$-axis could represent the effect of $\sim 5 \mathrm{~km} \mathrm{~s}^{-1}$ accuracy in the $\sigma_{V}$ dispersion of $30 \mathrm{~km} \mathrm{~s}^{-1}$. The offset $\delta E$ of $2 \%$ along the $E$-axis would leave a $1 \%$ offset that could not be explained just by a $1 \%$ offset $\delta V$ in $V$ (derived from the offset $\delta L_{z}$ along the $L_{z}$-axis). It may be conjectured (Fresneau et al. 2005) that we observe the transfer of angular momentum to energy $\delta E=\Omega_{p} \delta L_{z}$ by the spiral wave (Lynden-Bell \& Kalnajs 1972) where $\Omega_{p}$ is the angular velocity of the spiral pattern. We know that a relevant model for 
Table 4. Sydney Observatory Galactic Survey of N stars along galactic equator $l \in\left[272^{\circ}, 341^{\circ}\right], b \in\left[-7^{\circ}, 7^{\circ}\right]$, and galactic meridian $l=(330 \pm$ $5)^{\circ}, b \in\left[-64^{\circ},-7^{\circ}\right]$ covered by the Sydney Observatory Astrographic Program $\delta \in\left[-69^{\circ},-51^{\circ}\right]$ and compared to GSC version 1.2.

\begin{tabular}{ccc}
\hline \hline$\delta$ & $N$ & id \\
\hline$-52^{\circ}$ & 30143 & soap52 \\
$-53^{\circ}$ & 20177 & soap53 \\
$-54^{\circ}$ & 22509 & soap54 \\
$-55^{\circ}$ & 21715 & soap55 \\
$-56^{\circ}$ & 32791 & soap56 \\
$-57^{\circ}$ & 44386 & soap57 \\
$-58^{\circ}$ & 48484 & soap58 \\
$-59^{\circ}$ & 51795 & soap59 \\
$-60^{\circ}$ & 53464 & soap60 \\
$-61^{\circ}$ & 56523 & soap61 \\
$-62^{\circ}$ & 49495 & soap62 \\
$-63^{\circ}$ & 33792 & soap63 \\
$-64^{\circ}$ & 10058 & soap64 \\
$-68^{\circ}$ & 7864 & soap68 \\
\hline
\end{tabular}

a two-armed spiral pattern with constant Hamiltonian in the rotating system is $H \equiv \frac{1}{2}\left(u^{2}+V^{2}+w^{2}+\frac{L_{z}{ }^{2}}{R^{2}}\right)-\Phi+\Delta \Phi-\Omega_{p} L_{z}=$ $h$ (Jacobi constant). In this formulation, $\Delta \Phi$ is the perturbation of the axisymmetric component of the potential $\Phi$ due to spiral arms: $\Delta \Phi=\epsilon A(R) \cos [2 \varphi-\psi(R)]$ where $\epsilon=$ a measure of the strength of the spiral perturbation, $(R, \varphi)=$ stellar polar coordinates in the galactic plane, $A(R)=$ the density wave amplitude slowly varying with $R, \psi(R)=$ the density wave phase rapidly varying with $R$ (Contopoulos 1973).

The observations $\left(E, L_{z}\right)$ are fitted by a weighted least squares technique wit a $\chi^{2}$ level of significance of $10 \%$ and yield the following relations: $E=(193.80 \pm 018)-(2.803 \pm$ $0.009) L_{z}$ and $E=(192.67 \pm 0.06)-(2.618 \pm 0.003) L_{z}$. We derive from the previous adjustment $\Omega_{p} \simeq 16.4 \mathrm{~km} \mathrm{~s}^{-1} \mathrm{kpc}^{-1}$, close to the $17.1 \mathrm{~km} \mathrm{~s}^{-1} \mathrm{kpc}^{-1}$ value we previously found (Fresneau et al. 2005).

In view of these results, it is suggested that the p.m. perpendicular to the plane $\mu_{b}$ is purely due to random motions, while the observed p.m. in the plane $\mu_{l}$ is a line-of-sight integration of both the random motion and the systemic rotation that broadens the dispersion at a distance $R \mathrm{kpc}$ from the galactic centre as $\left\langle\mu_{l}{ }^{2}\right\rangle=\left\langle\mu_{b}{ }^{2}\right\rangle+\left\langle\left(\Omega_{p} \frac{R-R_{\odot}}{R_{\odot}}\right)^{2}\right\rangle$. A rotation speed of $\Omega_{p}=$ $16.4 \mathrm{~km} \mathrm{~s}^{-1} \mathrm{kpc}^{-1}$ agrees with the value found in our observations with $\frac{\left\langle\mu_{l}\right\rangle}{\left\langle\mu_{b}\right\rangle}=\frac{1}{3}$.

We provide in Table 4 the information to get the list of $4.8 \times$ $10^{5}$ stars used to trace the deflection of the velocity ellipsoid resulting from the crossing of the layer of $50 \mathrm{pc}$ thickness. For each star, a probability (from 0 to 1 ) is computed that shows the confidence to the fit to the inclination $\theta$ of the velocity vector $(V, W)$ with $\theta \propto z\left(z=\right.$ the height above the galactic plane, $\left.\frac{\partial \theta}{\partial z}=\frac{1}{2}{ }^{\circ} \mathrm{pc}^{-1}\right)$. The data are available on-line $e^{5}$ where each file contains the J2000 equatorial coordinates $(\alpha, \delta)$, J2000 equatorial components of the p.m. $\left(\mu_{\alpha} \cos \delta, \mu_{\delta}\right.$ in " $\left.\mathrm{yr}^{-1}\right), B$ and $V$ magnitudes, estimated parallax and its error $\left(\pi, \Delta \pi\right.$ in $\left.10^{-3 \prime \prime}\right)$ and probability (between 0 and 1$)$ for the tilt-term $\theta$ in the $(V, W)$ plane to follow the relation $\frac{\partial \theta}{\partial z}=\left(\frac{1}{2} \pm \frac{1}{4}\right)^{\circ} \mathrm{pc}^{-1}$.

\footnotetext{
${ }^{5}$ See ftp://cdsarc.u-strasbg.fr/incoming/fresneau/sogs
}

\section{Concluding remarks}

Rudimentary information (equatorial coordinates and apparent $B$-magnitude) provided by a magnitude-limited photographic survey down to $B=14.5$ is used to select a sample of $4.8 \times$ $10^{5}$ stars with significant p.m. $0.005 \leq \mu \leq 0.300^{\prime \prime} \mathrm{yr}^{-1}$ along the galactic equator $272^{\circ} \leq l \leq 341^{\circ},|b| \leq 7^{\circ}$ and along the galactic meridian $l=330^{\circ} \pm 5^{\circ},-64^{\circ} \leq b \leq-7^{\circ}$. The total p.m. $\mu$, is taken as as an indicator of distance when using the reduced proper motion $H_{B}=m_{B}+5+5 \log \mu$ to approximate an absolute magnitude $M_{B}=-3.76+0.711 H_{B}$ in the range from +2.0 to +8.4 with an error of $0.9 \mathrm{mag}$. This relation $M_{B}=-3.76+0.711 H_{B}$ is considered as fundamentally valid for stars with transverse velocity of order $45 \mathrm{~km} \mathrm{~s}^{-1}$, i.e. typical for F-G disk dwarfs.

The application of the Reduced Proper Motion relation on the data provided by SOGS provides a way to derive a statistical parallax $\pi$ and the analysis of the $(V, W)$ velocity components suggests a deflection of the velocity ellipsoid inclination $|\theta| \sim 20^{\circ}$ at a height $z= \pm 25 \mathrm{pc}$ over of the galactic plane. We suggest that the variation of the velocity ellipsoid parameters is the result of the crossing of the boundary layer between the Extreme and Thin disks of our Galaxy. We emphasize that we do not have any spectral characterization of the system of $4.8 \times 10^{5}$ stars, excepting an indication of approximate colour. The lack of spectral information prevents the search for kinematical stellar groups in the solar neighborhood (Elias et al. 2006; Famaey et al. 2005). Only disk crossing stars are tentatively identified by the tilt term $\theta$ of the projected velocity ellipsoid along the galactic equator. The observed spin rate modulus $\left|\frac{\partial \theta}{\partial z}\right| \sim\left(\frac{1}{2} \pm \frac{1}{4}\right)^{\circ} \mathrm{pc}^{-1}$ is interpreted as a scattering of disk crossing stars by ISM encounter in the galactic plane (Spitzer 1942).

Non-axisymmetric perturbations contribute to the tilt of the velocity ellipsoid since the galactic potential is the phenomenon that correlates the ellipsoid elements between them (Chandrasekhar 1940). In the present paper, a dynamical model of the Galaxy is not constructed that would be the exact solution for the Boltzmann equation incorporating both the tilt and the change in the shape of the velocity ellipsoid. Instead, an attempt is made to derive the surface mass density difference at the interface of a two-fluid system (a thin layer of gas+dust and a thick layer of stars) since our observations suggest a spin rate on the order of $\frac{1}{2}^{\circ} \mathrm{pc}^{-1}$ along the long axis of the velocity ellipsoid of stellar systems crossing the extreme thin disk. Since the behavior of the tilt term of the ellipsoidal distribution of stars in the space-velocity phase is being investigated, the p.m. and statistical parallax data derived in this work from 100 -year-old plates are a fundamental source of raw material for the optical study of the Galaxy kinematics in the greater solar neighborhood. We suggest that the boundary layer that we detect at $z= \pm 25 \mathrm{pc}$ is in relation to the large-scale dynamics (Chakrabarti et al. 2003; Olling \& Dehnen 2003) of the galactic extreme thin disk. The boundary layer processes on the small vertical scale $( \pm 25 \mathrm{pc})$ could be investigated by selecting the appropriate extreme disk crossing stars from SOGS and present epoch surveys (Girard et al. 2004; Gould \& Kollmeier 2004).

We suggest the following criteria suitable to the analysis of the data derived in SOGS:

- $\Delta x, \Delta y \leq 15$ microns for the use of stellar plate coordinates $x, y$.

- $\Delta \alpha, \Delta \delta \leq 1$ arcsec for the use of stellar equatorial coordinates $(\alpha, \delta)$.

- Total p.m. component $\mu \geq 0.015 \operatorname{arcsec} \cdot \mathrm{yr}^{-1}$ for high p.m. candidates with apparent $B$-magnitude $m \leq 15$. 
- $0.2 \leq B-V \leq 1.0$ to select F-G stellar candidates around $B-V=0.62$.

- $\frac{\Delta \pi}{\pi} \leq 30 \%$ for the analysis of the $(V, W)$ velocities derived from the statistical parallax $\pi$ and its estimated error $\Delta \pi$.

- The probability for a star to be considered as a disk crossing candidate should be larger than $\frac{1}{2}$.

The SOGS survey can be considered as an extension of the Tycho-2 survey to a fainter magnitude limit, with a similar accuracy in the p.m. determination and with an indication of a statistical parallax based on a correlation observed between the trigonometric parallax and the p.m. provided by Hipparcos (Narayanan \& Gould 1999) for stars up to a distance of $100 \mathrm{pc}$ (Dehnen 1998; Dehnen \& Binney 1998).

Acknowledgements. We thank the two referees for their help in improving the clarity of this paper. Our study has been supported by various Macquarie University research grants and by a French CNRS and British Royal Astronomical Society grant. We thank Mike Irwin from the Institute of Astronomy in Cambridge for letting us use the APM and François Ochsenbein from CDS for his advice in archiving the present results.

\section{References}

Babu, G. J., \& Feigelson, E. D. 1996, Astrostatistics (London: Chapman \& Hall) Bahcall, J. N., \& Soneira, R. M. 1980, ApJS, 44, 73

Bertin, G., \& Cava, A. 2006, A\&A, 459, 333

Bienaymé, O., Soubiran, C., Mishenina, T. V., et al. 2006, A\&A, 446, 933

Binney, J. 2005, MNRAS, 363, 937

Binney, J., \& Merrifield, M. 1998, Galactic Astronomy (Princeton, New Jersey: Princeton University Press)

Binney, J., Dehnen, W., \& Bertelli, G. 2000, MNRAS, 318, 658

Branham, R. L. 2004, A\&A, 421, 977

Carlberg, R. G., \& Innanen, K. A. 1987, AJ, 94, 666

Chakrabarti, S., Laughlin, G., \& Shu, F. H. 2003, ApJ, 596, 220

Chandraskhar, S. 1940, ApJ, 92, 441

Contopoulos, G. 1973, ApJ, 181, 657

Cuisinier, F., Buser, R., Acker, A., et al. 1994, A\&A, 285, 943

Dehnen, W. 1998, AJ, 115, 2384

Dehnen, W., \& Binney, J. 1998, MNRAS, 298, 387
Ducourant, C., Le Campion, J. F., Rapaport, M., et al. 2006, A\&A, 448, 1235 Edvarsson, B., Andersen, J., Gustafsson, B., et al. 1993, A\&A, 275, 101

Eichhorn, H., \& Williams, C. A. 1963, AJ, 68, 221

Elias, F., Alfaro, E. J., \& Cabrera-Caño, J. 2006, AJ, 132, 1052

Evans, D. W. 1992, MNRAS, 255, 521

Famaey, B., Jorissen, A., Luri, X., et al. 2005, A\&A, 430, 165

Fresneau, A. 1985, AJ, 90, 892

Fresneau, A. 1990, AJ, 100, 1223

Fresneau, A., Argyle, R. W., Marino, G., \& Messina, S. 2001, AJ, 121, 517

Fresneau, A., Vaughan, A. E., \& Argyle, R. W. 2003, AJ, 125, 1519

Fresneau, A., Vaughan, A. E., \& Argyle, R. W. 2005, AJ, 130, 2701

Girard, T. M., Dinescu, D. I., van Altena, W. F., et al. 2004, AJ, 127, 3060

Gould, A., \& Kollmeier, J. A. 2004, ApJS, 152, 103

Hanson, R. B., Klemola, A. R., Jones, B. F., \& Monet, D. G. 2004, AJ, 128, 1430

Høg, E., Fabricius, C., Makarov, V. V., et al. 2000, A\&A, 355, L27

Holmberg, J., \& Flynn, C. 2000, MNRAS, 313, 209

Jenkins, A., \& Binney, J. 1990, MNRAS, 245, 305

Johnson, D. R. H., \& Soderblom, D. R. 1987, AJ, 93, 864

Jones, E. M. 1972, ApJ, 173, 671

König, A. 1962, Astronomical Techniques, ed. W. A. Hiltner (Chicago: University of Chicago Press)

Lasker, B. M., Sturch, C. R., McLean, B. J., et al. 1990, AJ, 99, 2019

Lépine, S., Shara, M., \& Rich, R. M. 2002, AJ, 124, 1190

Lépine, S., Rich, R. M., \& Shara, M. M. 2003, AJ, 125, 1598

Lutz, T. E., \& Upgren, A. R. 1980, AJ, 85, 1390

Lynden-Bell, D., \& Kalnajs, A. D. 1972, MNRAS, 157, 1

May, A., \& Binney, J. 1986, MNRAS, 221, 857

Narayanan, V. K., \& Gould, A. 1999, ApJ, 523, 328

Olling, R. P., \& Dehnen, W. 2003, ApJ, 599, 275

Perryman, M. A. C., et al. 2007, The Hipparcos and Tycho catalogues, Vol. 1 (ESA SP-1200; Noodwijk: ESA)

Quillen, A. C., \& Minchev, I. 2005, AJ, 130, 576

Rapaport, M., Ducourant, C., Le Campion, J. F., et al. 2006, A\&A, 449, 435

Salim, S., \& Gould, A. 2002, ApJ, 575, L83

Sanner, J., Brunzendorf, J., Will, J.-M., \& Geffert, M. 2001, A\&A, 369, 511

Sellwood, J. A., \& Binney, J. J. 2002, MNRAS, 336, 785

Shu, F. 1969, ApJ, 158, 505

Spagna, A., Lattanzi, M. G., Lasker, B. M., et al. 1996, A\&A, 311, 758

Spitzer, L., Jr. 1942, ApJ, 95, 329

Steinmetz, M., Zwitter, T., Siebert, A., et al. 2006, AJ, 132, 1645

Turner, H. H. 1893, MNRAS, 54, 11

Urban, S. E., Corbin, T. E., Wycoff, G. L., et al. 1998, AJ, 115, 1212

Vyssotsky, A. N. 1954, AJ, 59, 52 